\title{
Review Article \\ Cyclin D1 G870A Polymorphism and Risk of Nasopharyngeal Carcinoma: A Meta-Analysis
}

\author{
Meng Li, ${ }^{1,2}$ Weijian Dai, ${ }^{3}$ and Huanqin Zhou ${ }^{1,2}$ \\ ${ }^{1}$ Department of Laboratory Medicine, Zhejiang Hospital, Hangzhou 310013, China \\ ${ }^{2}$ Zhejiang Provincial Key Lab of Geriatrics, Hangzhou 310013, China \\ ${ }^{3}$ Department of Laboratory Medicine, The First People's Hospital of Hangzhou, Hangzhou 310006, China
}

Correspondence should be addressed to Meng Li; lmbcwan@163.com

Received 2 August 2013; Accepted 1 September 2013

Academic Editors: J. M. Nesland and E. Tagliabue

Copyright (C) 2013 Meng Li et al. This is an open access article distributed under the Creative Commons Attribution License, which permits unrestricted use, distribution, and reproduction in any medium, provided the original work is properly cited.

\begin{abstract}
Recently, there have been a number of studies on the association between cyclin D1 G870A polymorphism and nasopharyngeal carcinoma risk. However, the results of previous reports remain controversial and ambiguous. Thus, we performed a meta-analysis to explore more precisely the association between cyclin D1 G870A polymorphism and the risk of nasopharyngeal carcinoma. No significant association was found between cyclin D1 G870A polymorphism and nasopharyngeal carcinoma risk in total population analysis. In the subgroup meta-analysis by ethnicity, a negative association was shown in Caucasian subgroup, and no significant association in any genetic models among Asians was observed. In summary, positive results have been shown on the search for polymorphic variants influencing the risk of NPC. This meta-analysis provides evidence of the association between CCND1 G870A polymorphism and NPC risk, supporting the hypothesis that CCND1 870A allele probably acts as an important NPC protective factor in Caucasians but not in Asians. Since the results of our meta-analysis are preliminary and may be biased by the relatively small number of subjects, they still need to be validated by well-designed studies using larger samples in the future.
\end{abstract}

\section{Introduction}

Nasopharyngeal carcinoma (NPC) is a malignant epithelial cancer with a strikingly geographic and ethnic distribution. The incidence of NPC is higher in Southeast Asia and Africa, but lower among Caucasians in North America and Europe. Epidemiological studies and experimental researches have implicated genetic susceptibility, Epstein-Barr viral (EBV) infection, and environmental factors in the specific and multifactorial etiology of NPC [1]. In spite of many individuals being exposed to EBV infection and environmental risk factors including extensive tobacco and alcohol consumption, only a small population can be clinically diagnosed with NPC, which suggests that individual genetic susceptibility might play a more important role in the carcinogenic mechanisms of NPC. However, the precise genetic alterations during NPC development are still unclear.

Cyclin D1, encoded by CCND1 gene, plays a critical role in the transition from G1 to $S$ phase of the cell cycle during cell division. CCND1 amplification and protein overexpression were detected (described) in NPC patients [2]. The activation of cyclin D1 participates in tumorigenesis [3], local tumor recurrence, and poor prognosis of NPC [2]. CCND1 G870A (rs603965) polymorphism is common in a variety of human tumors, including breast cancer, lung cancer, gastric cancer, gynecological cancer, blood-related cancer, and colorectal cancer (NPC) [4, 5]. Although CCND1 G870A polymorphism is a silent mutation (Pro241Pro), 870A allele results in an alternatively spliced transcript of CCND1 (transcript b), which has been shown to have a longer half-life than the $G$ allele (transcript a) encoded protein. It suggests that CCND1 $870 \mathrm{~A}$ allele is more likely to contribute to cancer development through promoting the transition between G1 and S phases [6]. To date, although a few studies have linked the CCND1 G870A polymorphism to the increased NPC risk, the results remain controversial.

Considering a single study may be insufficient to identify the effect of CCND1 G870A polymorphism on NPC, and 
the published results have been controversial. We therefore performed a meta-analysis to assess the association between CCND1 G870A polymorphism and NPC susceptibility.

\section{Methods}

2.1. The Literature Search Strategy. We searched the literature databases including PubMed, ISI Web of Science, Chinese National Knowledge Infrastructure (CNKI), and Google Scholar (up to November 08, 2012). The search strategy for identifying all possible studies involved usage of the following keywords: "cyclin D1 or CCND1" and "polymorphism or variant or genotype or SNP" and "nasopharyngeal carcinoma or nasopharyngeal cancer or squamous cell cancer of the head and neck or head and neck cancer". All related studies without language limitation were included. The reference lists of the additional articles reporting the association between CCND1 G870A polymorphism and NPC were hand searched.

2.2. Eligible Studies and Data Extraction. All the studies included in the meta-analysis met the following inclusion criteria: (1) original papers associated cyclin D1 G870A polymorphism with NPC; (2) case control or cohort design was used, and (3) genotype distribution information, odds ratio (OR) with its $95 \%$ confidence interval (CIs), and $P$ value were provided. The major exclusion criteria were (1) duplicate data, (2) case-only studies, (3) review articles, and (4) studies with association between cyclin D1 G870A polymorphism and head and neck cancer, which just described the number and anatomical distribution of tumors without specifically showing the genotype distribution information of each tumor, including NPC.

Data extraction for compliance with the inclusion/exclusion criteria was performed independently by two reviewers. Disagreements were resolved by further discussion among all authors. For each included study, the following information was extracted according to a fixed protocol: (1) name of the first author; (2) year of publication; (3) country; (4) ethnicity; (5) genotyping method; (6) genotype distribution in cases and controls; (7) $P$ value for Hardy-Weinberg equilibrium (HWE) test in controls.

2.3. Statistical Analysis. The association between cyclin D1 G870A polymorphism and NPC was estimated by calculating pooled odds ratio (OR) and 95\% confidence interval (CI) under a homozygote comparison model (AA versus GG), a heterozygote comparison model (GA versus GG), and a dominant model (AA + GA versus GG), respectively. The significance of pooled OR was determined by $Z$ test. Cochran's chi-square-based Q statistic test was performed to evaluate the possible heterogeneity among individual studies. Pooled ORs were calculated according to a fixed model (DerSimonian-Laird method) or a random model (MantelHaenszel method) in the absence $(P>0.10)$ or presence $(P \leq$ $0.10)$ of heterogeneity. Heterogeneity was explored using the subgroup analysis of ethnicity (Asians and Caucasians). Publication bias was assessed statistically by Egger's test and Begger's test. The Hardy-Weinberg equilibrium (HWE) was

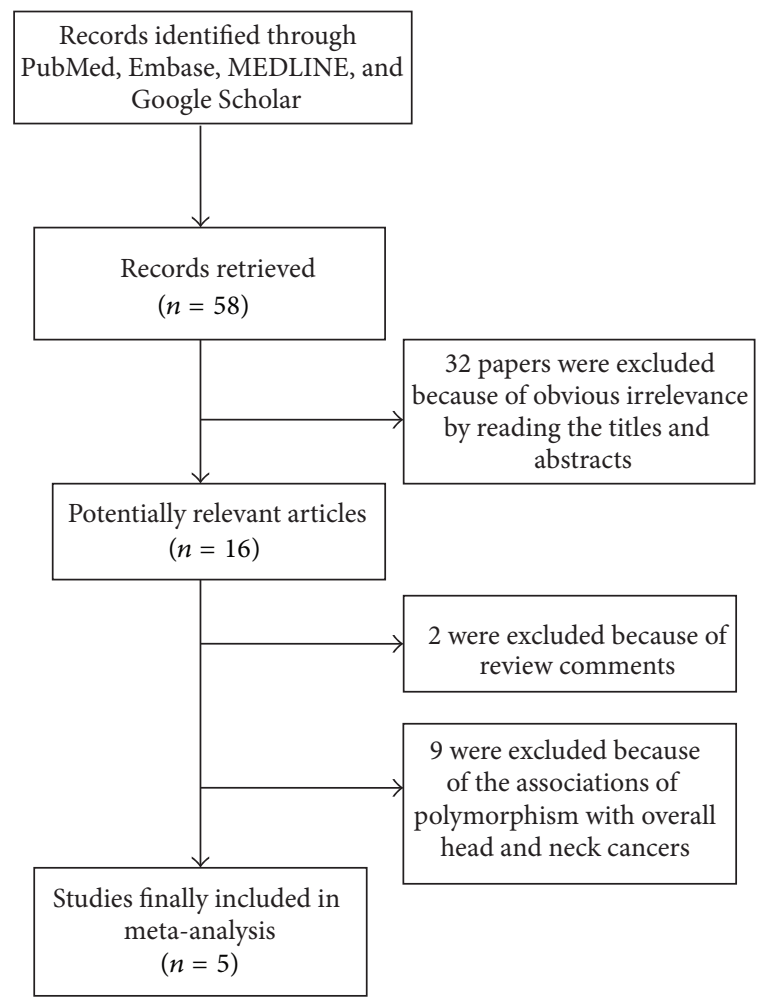

FIGURE 1: Flow chart of study selection based on the inclusion and exclusion criteria.

determined using the chi-square test in control groups. All the data analyses were performed using STATA software version 11.0 (StataCorp LP, College Station, Texas, USA). All $P$ values were calculated with two-sided analysis, and a $P$ value less than 0.05 was considered as statistical significance.

\section{Results}

3.1. Study Selection. As shown in Figure 1, through the literature search, we have identified 58 potentially relevant papers. After a careful review, 32 papers were excluded because of obvious irrelevance by evaluating the contents of abstracts. In addition, two reviews [5, 7] and nine papers [8-16] which assessed the association between polymorphism and overall head and neck cancers were excluded. Finally, five studies (including 679 cases and 973 controls) studying the cyclin D1 G870A polymorphisms [17-21] met the inclusion criteria and were selected for the meta-analysis.

3.2. Characteristics of the Studies. Characteristics of the studies included in this meta-analysis were presented in Table 1. All studies were case-controlled. Of these 5 studies, 4 used polymerase chain reaction-restriction fragment length polymorphism (PCR-RFLP) and 1 used denaturing highperformance liquid chromatography (DHPLC). All studies were carried out in the mainland, Taiwan of China, and Portugal. Three studies were on Asians, and two studies were on Caucasians. Studies being carried out in the mainland and Taiwan of China were grouped to the Asian subgroup, 
TABLE 1: Characteristics of the studies included in the meta-analysis.

\begin{tabular}{|c|c|c|c|c|c|c|c|c|c|c|c|c|}
\hline \multirow{3}{*}{ First author } & \multirow{3}{*}{ Year } & \multirow{3}{*}{ Country } & \multirow{3}{*}{ Ethnicity } & \multirow{3}{*}{ Genotyping method } & \multicolumn{6}{|c|}{ Genotype distribution } & \multirow{3}{*}{$P$ for $\mathrm{HWE}^{\mathrm{a}}$} & \multirow{3}{*}{ References } \\
\hline & & & & & \multicolumn{3}{|c|}{ Case } & \multicolumn{3}{|c|}{ Control } & & \\
\hline & & & & & GG & GA & AA & GG & GA & AA & & \\
\hline Catarino & 2008 & Portugal & European & PCR-RFLP & 23 & 40 & 21 & 40 & 138 & 69 & 0.036 & [17] \\
\hline Catarino & 2006 & Portugal & European & PCR-RFLP & 26 & 42 & 26 & 28 & 105 & 54 & 0.047 & {$[18]$} \\
\hline Deng & 2002 & China (mainland) & Asian & DHPLC & 19 & 48 & 17 & 14 & 42 & 35 & 0.811 & {$[19]$} \\
\hline Shih & 2012 & China (Taiwan) & Asian & PCR-RFLP & 23 & 86 & 67 & 28 & 105 & 43 & 0.007 & {$[20]$} \\
\hline Sui & 2009 & China (mainland) & Asian & PCR-RFLP & 60 & 110 & 71 & 115 & 124 & 33 & 0.962 & {$[21]$} \\
\hline
\end{tabular}

${ }^{\mathrm{a}} \mathrm{HWE}$ in controls.

TABLE 2: Meta-analysis of the association between cyclin D1 G807A polymorphism and nasopharyngeal cancer risk.

\begin{tabular}{|c|c|c|c|c|c|c|}
\hline \multirow{2}{*}{ Comparisons } & \multirow{2}{*}{ Odds ratio } & \multirow{2}{*}{$95 \%$ confidence interval } & \multirow{2}{*}{$P$ value } & \multicolumn{2}{|c|}{ Heterogeneity } & \multirow{2}{*}{ Effects model } \\
\hline & & & & $I^{2}$ & $P$ value & \\
\hline A versus $G$ & 1.010 & $0.628-1.622$ & 0.969 & $90.5 \%$ & 0.000 & Random \\
\hline Asians & 1.221 & $0.647-2.304$ & 0.538 & $91.7 \%$ & 0.000 & \\
\hline Caucasians & 0.754 & $0.589-0.967$ & 0.026 & $0.0 \%$ & 0.989 & \\
\hline AA versus GG & 0.976 & $0.368-2.592$ & 0.961 & $90.2 \%$ & 0.000 & Random \\
\hline Asians & 1.475 & $0.407-5.345$ & 0.554 & $90.7 \%$ & 0.000 & \\
\hline Caucasians & 0.524 & $0.317-0.865$ & 0.011 & $0.0 \%$ & 0.968 & \\
\hline GA versus GG & 0.811 & $0.460-1.429$ & 0.469 & $77.6 \%$ & 0.001 & Random \\
\hline Asians & 1.236 & $0.791-1.913$ & 0.353 & $42.6 \%$ & 0.175 & \\
\hline Caucasians & 0.467 & $0.299-0.730$ & 0.001 & $0.0 \%$ & 0.730 & \\
\hline $\mathrm{GA}+\mathrm{AA}$ versus $\mathrm{GG}$ & 0.856 & $0.430-1.707$ & 0.660 & $86.6 \%$ & 0.000 & Random \\
\hline Asians & 1.277 & $0.631-2.584$ & 0.497 & $78.4 \%$ & 0.010 & \\
\hline Caucasians & 0.487 & $0.319-0.741$ & 0.001 & $0.0 \%$ & 0.804 & \\
\hline
\end{tabular}

while the others were grouped to the Caucasian subgroup. The distribution of genotypes in the controls was consistent with Hardy-Weinberg equilibrium $(P>0.05)$ in all studies except for three studies (Catarino et al. [17], $P=0.037$; Shih et al. [20], $P=0.007$; Catarino et al. [18], $P=0.047)$.

3.3. Quantitative Data Synthesis. The results on the association between cyclin D1 G870A polymorphism and NPC risk and the heterogeneity test were shown in Table 2 . The combined results based on all studies showed that the variant genotypes were not associated with the increased NPC risk in different genetic models $(\mathrm{OR}=1.010,95 \% \mathrm{CI}=0.628-1.622$ for A versus $\mathrm{G}, P=0.969 ; \mathrm{OR}=0.976,95 \% \mathrm{CI}=0.368-2.592$ for homozygote comparison model AA versus GG, $P=0.961$; $\mathrm{OR}=0.811,95 \% \mathrm{CI}=0.460-1.429$ for heterozygote comparison model GA versus GG, $P=0.469$; OR $=0.856,95 \%$ $\mathrm{CI}=0.430-1.707$ for dominant model GA + AA versus $\mathrm{GG}$, $P=0.660$ ) (Figures 2, 3, 4, and 5). In the subgroup analysis by ethnicity, the results revealed a significant association between the cyclin D1 G870A polymorphism and NPC in Caucasian population (A versus G: OR $=0.754,95 \%$ $\mathrm{CI}=0.589-0.967, P=0.026, P_{\text {het }}=0.989$; homozygote comparison model AA versus GG: OR $=0.524,95 \%$ $\mathrm{CI}=0.317-0.865, P=0.011, P_{\text {het }}=0.968$; heterozygote comparison model GA versus GG: OR $=0.467,95 \% \mathrm{CI}=$ $0.299-0.730, P=0.001, P_{\text {het }}=0.730$; dominant model GA $+\mathrm{AA}$ versus GG: $\mathrm{OR}=0.487,95 \% \mathrm{CI}=0.319-0.741$,
$\left.P=0.001, P_{\text {het }}=0.804\right)$. In contrast, no such significant association in any genetic models was observed in Asians (A versus $\mathrm{G}$ : $\mathrm{OR}=1.221,95 \% \mathrm{CI}=0.647-2.304, P=0.538$; homozygote comparison model AA versus GG: $\mathrm{OR}=1.475$, 95\% CI $=0.407-5.345, P=0.554$; heterozygote comparison model GA versus GG: $\mathrm{OR}=1.236,95 \% \mathrm{CI}=0.791-1.913$, $P=0.554$; dominant model GA + AA versus GG: OR = $1.277,95 \% \mathrm{CI}=0.631-2.584, P=0.497)$.

3.4. Tests of Heterogeneity. Statistically significant heterogeneity was observed in trials using the following analyses with $\mathrm{Q}$ statistic tests (A versus G: $P=0.000, I^{2}=90.5 \%$; homozygote comparison model AA versus GG: $P=0.000$, $I^{2}=90.2 \%$; heterozygote comparison model GA versus GG: $P=0.001, I^{2}=77.6 \%$; dominant model GA + AA versus GG: $P=0.000, I^{2}=86.6 \%$ ) (Table 2 ) and employing the randomeffects model.

3.5. Publication Bias. Egger's test and Beggar's test were performed to assess publication bias. Analysis using the Egger weighted regression method did not indicate publication bias for two of the four genetic models (heterozygote comparison model GA versus GG: $P=0.143$; dominant model GA + AA versus GG: $P=0.082)$, but indicated evidence for publication bias for the other two genetic models (A versus $\mathrm{G}$ : $P=0.007$; homozygote comparison model AA versus GG: $P=0.027$ ). 


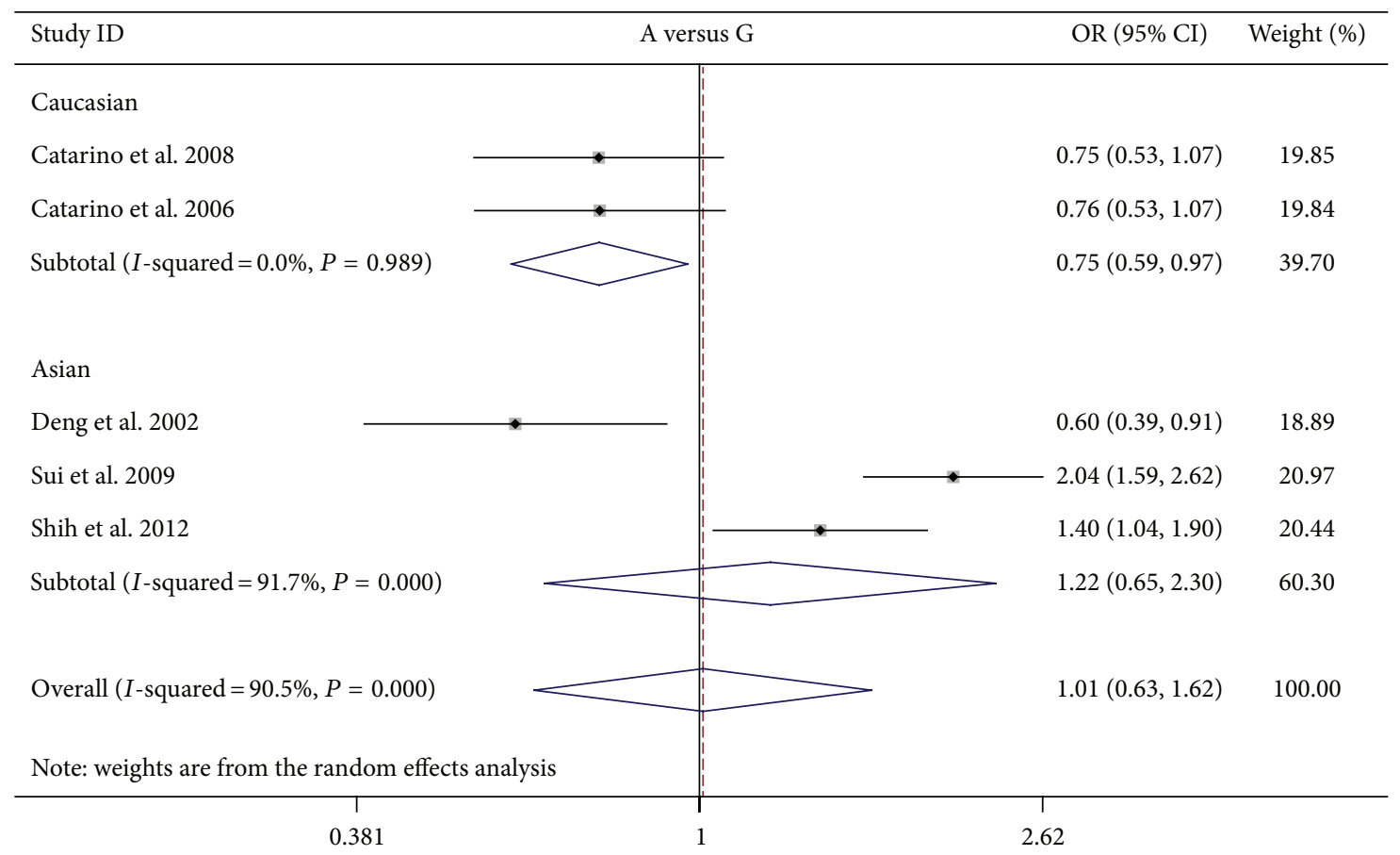

FIGURE 2: Forest plots of cyclin D1 G870A polymorphism in nasopharyngeal carcinoma versus normal control and subgroup analyses for A genotype compared with G genotype. The squares and horizontal lines correspond to the study specific OR and 95\% CI. The area of the squares reflects the weight (inverse of the variance). The diamond represents the summary OR and 95\% CI. OR: odds ratio.

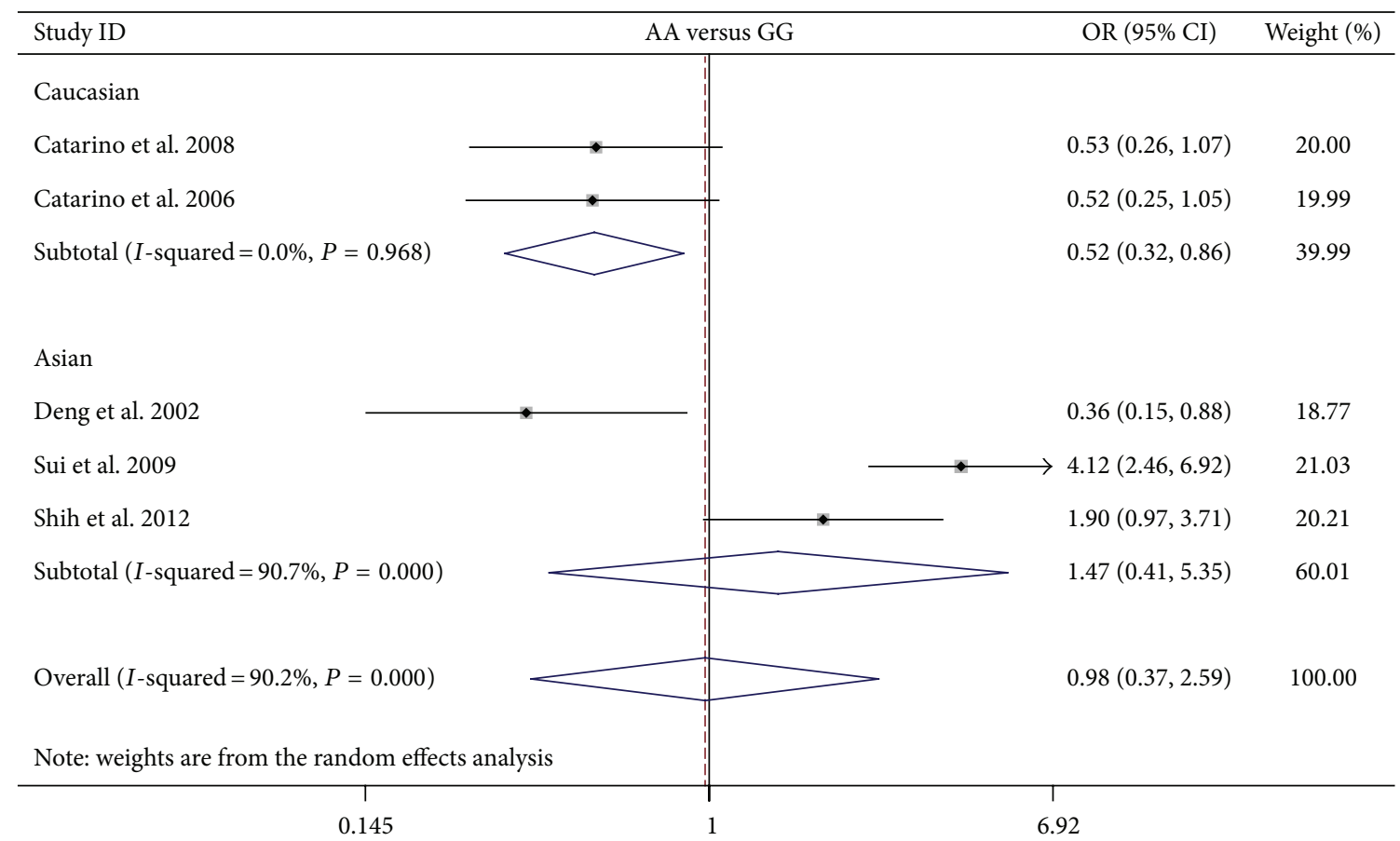

FIGURE 3: Forest plots of cyclin D1 G870A polymorphism in nasopharyngeal carcinoma versus normal control and subgroup analyses for AA genotype compared with GG genotype. 
TABLE 3: Publication bias test for cyclin D1 G807A polymorphism.

\begin{tabular}{lcccc}
\hline Comparisons & & Egger's test & Beggar's test $P$ value \\
& Coefficient & $P$ value & $95 \%$ CI & 0.086 \\
A versus G & -15.91 & 0.007 & $-23.44--8.39$ & 0.027 \\
AA versus GG & -14.52 & 0.037 & $-27.36--1.68$ & 0.221 \\
GA versus GG & -6.03 & 0.143 & $-15.77-3.71$ & 0.462 \\
GA + AA versus GG & -8.39 & 0.082 & $-18.76-1.96$ & \\
\hline
\end{tabular}

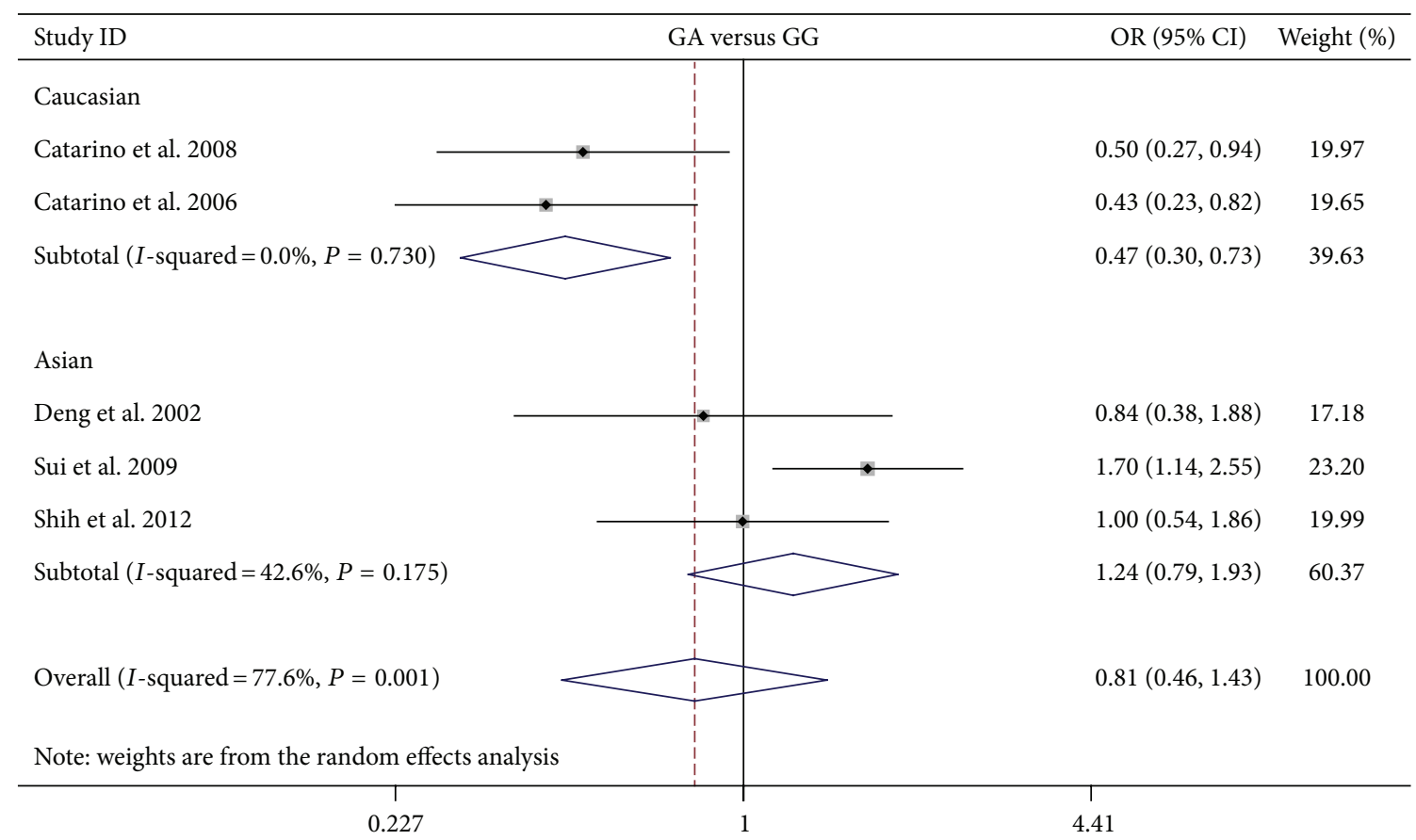

FIGURE 4: Forest plots of cyclin D1 G870A polymorphism in nasopharyngeal carcinoma versus normal control and subgroup analyses for GA genotype compared with GG genotype.

Beggar's rank correlation showed no evidence for publication bias for three of the four genetic models (A versus G: $P=$ 0.086 ; heterozygote comparison model GA versus GG: $P=$ 0.221; dominant model GA + AA versus GG: $P=0.462)$ but indicated publication bias for the homozygote comparison model AA versus GG $(P=0.027)$ (Table 3$)$.

\section{Discussion}

Cell cycle regulation plays an important role in the development of cancer by influencing cell proliferation, differentiation, and apoptosis [22]. The CCND1 gene encodes a key cell cycle regulatory protein, cyclin D1, which regulates transition from G1 to S phase during cell division. Cyclin D1 has been recognized as a promising biomarker for predicting tumor behavior [23]. In recent years, the common functional polymorphism, G870A in the gene cyclinD1, has been widely studied as a possible low-penetrant susceptibility allele for a variety of cancers. A number of studies found that the $G$ allele seems to be a protective factor in hepatocellular carcinoma [24], laryngeal [25], breast [26], colorectal [27, 28], and bladder tumors [29]. But several controversial findings reported that the G allele was a risky factor for oral [30] and colorectal cancer [31] or was not associated with various types of cancer [32-36]. Since conflicting results among studies or ethnic groups have been reported, it is necessary to make a quantitative and summarized evaluation of possible association between cyclin D1 G870A polymorphism and risk of cancer.

Deng et al. found that cyclin D1 G870A polymorphism was associated with the susceptibility to NPC, because the GG and AG genotypes in NPC patients were significantly higher than those in normal controls [19]. But Shih et al. reported that the G allele of CCND1 G870A seemed to be a protective factor for NPC in Taiwan of China [20]. Catarino et al. also reported that individuals carrying the CCND1 GG genotype had increased risk for the development of NPC [18]. Therefore, it is worthy to make a meta-analysis to evaluate the interrelationship between cyclin D1 G870A polymorphism and NPC. The current meta-analysis summarized the results from 5 case-controlled studies on the association between the CCND1 G870A polymorphism and NPC risk. A total of 679 cases and 973 controls were included. Although we found no significant risk of NPC associated with the CCND1 G870A polymorphism based on total population, 


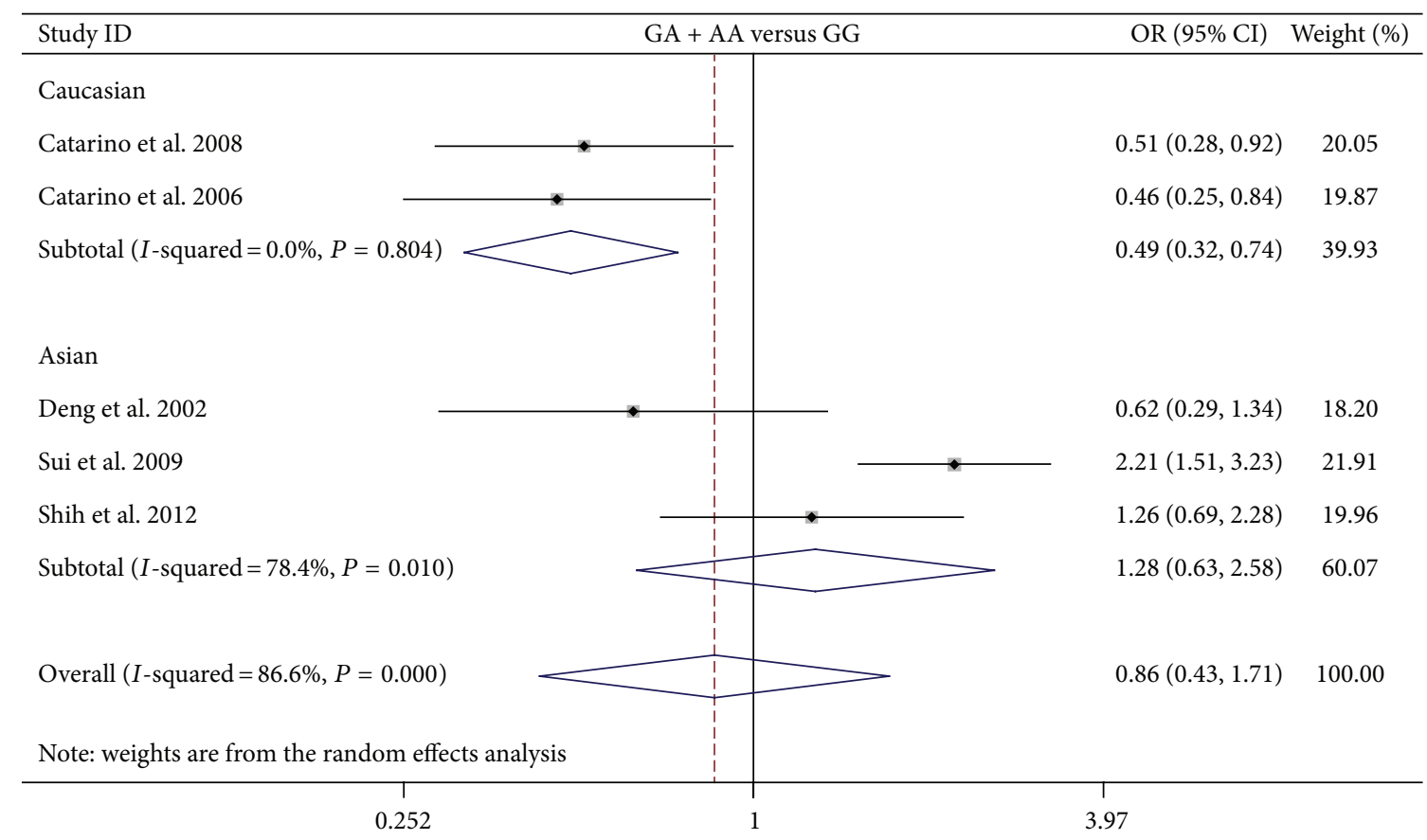

FIGURE 5: Forest plots of cyclin D1 G870A polymorphism in nasopharyngeal carcinoma versus normal control and subgroup analyses for AA + GA genotype compared with GG genotype.

significant association was found in Caucasian population in the subgroup analysis by ethnicity.

In the subgroup meta-analysis based on ethnicity, compared with G allele, a significantly decreased risk of NPC was associated with A allele; compared with GG genotype, a significantly decreased risk of NPC was associated with AA genotype, GA genotype, and combined AA/GA genotype in the Caucasian subgroup. Further investigations on a larger scale on Caucasian population may be needed to confirm this result. In the Asian subgroup, no significant association was found in the different genetic models. Our results indicate that ethnicity may be a critical factor affecting effects of the polymorphic alleles on susceptibility to NPC.

Despite these advantages, some limitations in the current meta-analysis should be considered with caution. Firstly, the controls were not uniformly defined. Although most control groups were selected from healthy populations, some might have a benign disease. Therefore, there is a lack of proper matching, and the results are based on unadjusted estimates. Secondly, the analysis did not consider gene-gene and gene-environment interactions due to the lack of sufficient data. Thirdly, environmental and lifestyle-related factors may influence the results of this analysis. A more precise analysis with individual data available might be considered, which could allow an adjustment estimate by sex, age, body weight, and lifestyle such as smoking and alcohol drinking. Fourthly, there is evidence of publication bias in the formal evaluation used in this study. The results of our meta-analysis may be biased by the relatively small number of subjects. Therefore, our conclusion still needs to be validated by well-designed studies with larger sample size in the future. Finally, and most importantly, whether the CCND1 G870A polymorphism is independently predictive of cancer risk remains controversial.

In summary, positive results have been shown in the search for polymorphic variants influencing the risk of NPC. This meta-analysis provides evidence of the association between CCND1 G870A polymorphism and NPC risk, supporting the hypothesis that CCND1 870A allele probably acts as an important NPC protective factor in Caucasians, but not in Asians.

\section{Conflict of Interests}

The authors declare that there is no conflict of interests regarding the publication of this paper.

\section{Acknowledgments}

This work was supported by grants from the National Science Foundation of China (Grant no. 81101491), Natural Science Foundation of Zhejiang Province of China (Grant no. Y2110843), and Zhejiang medical and health science and technology program (Grant no. 2012KYB153).

\section{References}

[1] E. T. Chang and H. Adami, "The enigmatic epidemiology of nasopharyngeal carcinoma," Cancer Epidemiology Biomarkers and Prevention, vol. 15, no. 10, pp. 1765-1777, 2006.

[2] C. F. Hwang, C. L. Cho, C. C. Huang et al., "Loss of cyclin D1 and p16 expression correlates with local recurrence in nasopharyngeal carcinoma following radiotherapy," Annals of Oncology, vol. 13, no. 8, pp. 1246-1251, 2002. 
[3] A. B. Hui, Y. Y. Or, H. Takano et al., "Array-based comparative genomic hybridization analysis identified cyclin D1 as a target oncogene at 11q13.3 in nasopharyngeal carcinoma," Cancer Research, vol. 65, no. 18, pp. 8125-8133, 2005.

[4] K. E. Knudsen, J. A. Diehl, C. A. Haiman, and E. S. Knudsen, "Cyclin D1: polymorphism, aberrant splicing and cancer risk," Oncogene, vol. 25, no. 11, pp. 1620-1628, 2006.

[5] N. Pabalan, B. Bapat, L. Sung, H. Jarjanazi, O. FranciscoPabalan, and H. Ozcelik, "Cyclin D1 Pro241Pro (CCND1G870A) polymorphism is associated with increased cancer risk in human populations: a meta-analysis," Cancer Epidemiology Biomarkers and Prevention, vol. 17, no. 10, pp. 2773-2781, 2008.

[6] D. A. Solomon, Y. Wang, S. R. Fox et al., "Cyclin D1 splice variants. Differential effects on localization, RB phosphorylation, and cellular trans-formation," The Journal of Biological Chemistry, vol. 278, no. 32, pp. 30339-30347, 2003.

[7] C. Tang, Z. Wang, J. Yu, Y. Wu, Z. Zhu, and N. Chen, "CCND1 G870A polymorphism and risk for head and neck cancer: a meta-analysis," Medical Oncology, vol. 28, no. 4, pp. 1319-1324, 2011.

[8] A. K. Azad, I. Bairati, E. Samson et al., "Validation of genetic sequence variants as prognostic factors in early-stage head and neck squamous cell cancer survival," Clinical Cancer Research, vol. 18, no. 1, pp. 196-206, 2012.

[9] E. Henriksson, E. Kjellén, B. Baldetorp et al., "Comparison of cisplatin sensitivity and the 18F fluoro-2-deoxy 2 glucose uptake with proliferation parameters and gene expression in squamous cell carcinoma cell lines of the head and neck," Journal of Experimental and Clinical Cancer Research, vol. 28, no. 1, article 17, 2009

[10] S. L. Holley, C. Matthias, V. Jahnke, A. A. Fryer, R. C. Strange, and P. R. Hoban, "Association of cyclin D1 polymorphism with increased susceptibility to oral squamous cell carcinoma," Oral Oncology, vol. 41, no. 2, pp. 156-160, 2005.

[11] J. Hopkins, D. W. Cescon, D. Tse et al., "Genetic polymorphisms and head and neck cancer outcomes: a review," Cancer Epidemiology Biomarkers and Prevention, vol. 17, no. 3, pp. 490-499, 2008.

[12] K. Jelonek, A. Gdowicz-Kłosok, M. Pietrowska et al., "Association between single-nucleotide polymorphisms of selected genes involved in the response to DNA damage and risk of colon, head and neck, and breast cancers in a Polish population," Journal of Applied Genetics, vol. 51, no. 3, pp. 343-352, 2010.

[13] C. Matthias, U. Harréus, and R. Strange, "Influential factors on tumor recurrence in head and neck cancer patients," European Archives of Oto-Rhino-Laryngology, vol. 263, no. 1, pp. 37-42, 2006.

[14] A. F. Olshan, M. C. Weissler, H. Pei et al., "Alterations of the p16 gene in head and neck cancer: frequency and association with p53, PRAD-1 and HPV," Oncogene, vol. 14, no. 7, pp. 811818, 1997.

[15] M. Sabir, R. M. Baig, I. Mahjabeen, and M. A. Kayani, "Significance of cyclin D1 polymorphisms in patients with head and neck cancer," International Journal of Biological Markers, vol. 28, no. 1, pp. 49-55, 2013.

[16] S. Zhong, T. Nukui, S. Buch et al., "Effects of ERCC2 Lys751Gln (A35931C) and CCND1 (G870A) polymorphism on outcome of advanced-stage squamous cell carcinoma of the head and neck are treatment dependent," Cancer Epidemiology Biomarkers and Prevention, vol. 20, no. 11, pp. 2429-2437, 2011.

[17] R. Catarino, D. Pereira, E. Breda et al., "Oncogenic virusassociated neoplasia: a role for cyclin D1 genotypes influencing the age of onset of disease?" Biochemical and Biophysical Research Communications, vol. 370, no. 1, pp. 118-122, 2008.

[18] R. J. Catarino, E. Breda, V. Coelho et al., "Association of the A870G cyclin D1 gene polymorphism with genetic susceptibility to nasopharyngeal carcinoma," Head and Neck, vol. 28, no. 7, pp. 603-608, 2006.

[19] L. Deng, X. R. Zhao, K. F. Pan et al., "Cyclin D1 polymorphism and the susceptibility to NPC using DHPLC," Acta Biochimica et Biophysica Sinica, vol. 34, no. 1, pp. 16-20, 2002.

[20] L. C. Shih, C. W. Tsai, M. H. Tsai et al., "Association of cyclin D1 genotypes with nasopharyngeal carcinoma risk," Anticancer Research, vol. 32, no. 3, pp. 1093-1098, 2012.

[21] J. Sui, W. Gao, X. J. Li et al., "Cyclin D1 gene G870A polymorphism and susceptibility to nasopharyngeal carcinoma," Zhonghua Er Bi Yan Hou Tou Jing Wai Ke Za Zhi, vol. 44, no. 3, pp. 227-231, 2009.

[22] G. I. Evan and K. H. Vousden, "Proliferation, cell cycle and apoptosis in cancer," Nature, vol. 411, no. 6835, pp. 342-348, 2001.

[23] A. N. Salesiotis and K. J. Cullen, "Molecular markers predictive of response and prognosis in the patient with advanced squamous cell carcinoma of the head and neck: evolution of a model beyond TNM staging," Current Opinion in Oncology, vol. 12, no. 3, pp. 229-239, 2000.

[24] H. Akkiz, S. Bayram, A. Bekar, E. Akgöllü, and B. Özdil, “Cyclin D1 G870A polymorphism is associated with an increased risk of hepatocellular carcinoma in the Turkish population: casecontrol study," Cancer Epidemiology, vol. 34, no. 3, pp. 298-302, 2010.

[25] M. Rydzanicz, P. Golusinski, D. Mielcarek-Kuchta, W. Golusinski, and K. Szyfter, "Cyclin D1 gene (CCND1) polymorphism and the risk of squamous cell carcinoma of the larynx," European Archives of Oto-Rhino-Laryngology, vol. 263, no. 1, pp. 43-48, 2006.

[26] E. Canbay, I. Y. Eraltan, C. Ali et al., "CCND1 and CDKN1B polymorphisms and risk of breast cancer," Anticancer Research, vol. 30, no. 7, pp. 3093-3098, 2010.

[27] F. Grünhage, M. Jungck, C. Lamberti et al., "Association of familial colorectal cancer with variants in the E-cadherin (CDH1) and cyclin D1 (CCND1) genes," International Journal of Colorectal Disease, vol. 23, no. 2, pp. 147-154, 2008.

[28] J. Jiang, J. Wang, S. Suzuki et al., "Elevated risk of colorectal cancer associated with the AA genotype of the cyclin D1 A870G polymorphism in an Indian population," Journal of Cancer Research and Clinical Oncology, vol. 132, no. 3, pp. 193-199, 2006.

[29] L. Yuan, X. Gu, J. Shao et al., "Cyclin D1 G870A polymorphism is associated with risk and clinicopathologic characteristics of bladder cancer," DNA and Cell Biology, vol. 29, no. 10, pp. 611617, 2010.

[30] K. M. Sathyan, K. R. Nalinakumari, T. Abraham, and S. Kannan, "CCND1 polymorphisms (A870G and C1722G) modulate its protein expression and survival in oral carcinoma," Oral Oncology, vol. 44, no. 7, pp. 689-697, 2008.

[31] I. Yaylim-Eraltan, S. Arikan, Y. Yildiz et al., "The influence of cyclin D1 A870G polymorphism on colorectal cancer risk and prognosis in a Turkish population," Anticancer Research, vol. 30, no. 7, pp. 2875-2880, 2010.

[32] R. Gangwar and R. D. Mittal, "Association of selected variants in genes involved in cell cycle and apoptosis with bladder cancer risk in north Indian population," DNA and Cell Biology, vol. 29, no. 7, pp. 349-356, 2010. 
[33] C. C. Gomes, S. N. Drummond, A. L. S. Guimarães, C. I. Andrade, R. A. Mesquita, and R. S. Gomez, "P21/WAF1 and cyclin D1 variants and oral squamous cell carcinoma," Journal of Oral Pathology and Medicine, vol. 37, no. 3, pp. 151-156, 2008.

[34] M. Jain, S. Kumar, R. Upadhyay et al., "Influence of apoptosis (BCL2, FAS), cell cycle (CCND1) and growth factor (EGF, EGFR) genetic polymorphisms on survival outcome: an exploratory study in squamous cell esophageal cancer," Cancer Biology and Therapy, vol. 6, no. 10, pp. 1553-1558, 2007.

[35] K. M. Sathyan, K. R. Nalinakumari, T. Abraham, and S. Kannan, "Influence of single nucleotide polymorphisms in H-Ras and cyclin D1 genes on oral cancer susceptibility," Oral Oncology, vol. 42, no. 6, pp. 607-613, 2006.

[36] K. Satinder, S. R. Chander, K. Pushpinder, G. Indu, and J. Veena, "Cyclin D1 (G870A) polymorphism and risk of cervix cancer: a case control study in north Indian population," Molecular and Cellular Biochemistry, vol. 315, no. 1-2, pp. 151-157, 2008. 


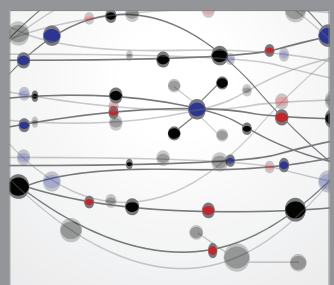

The Scientific World Journal
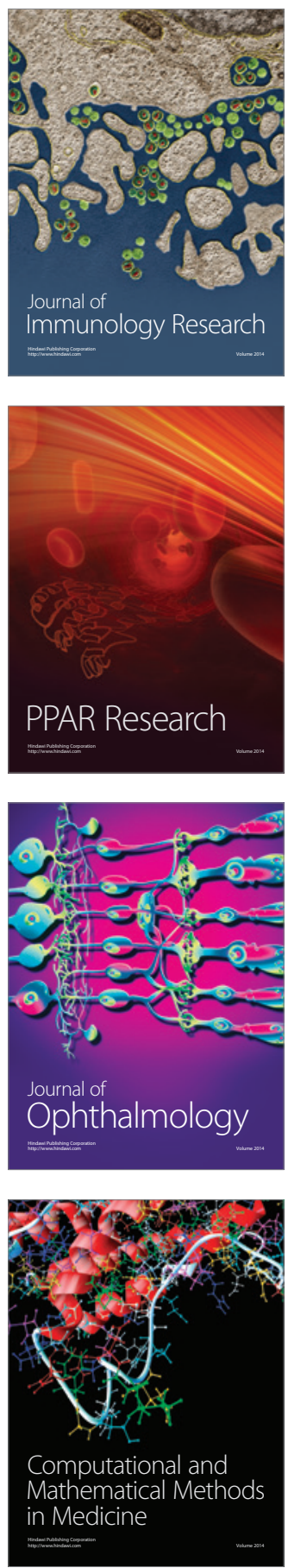

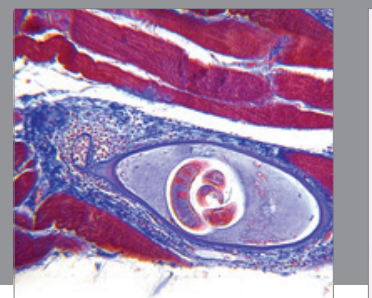

Gastroenterology

Research and Practice
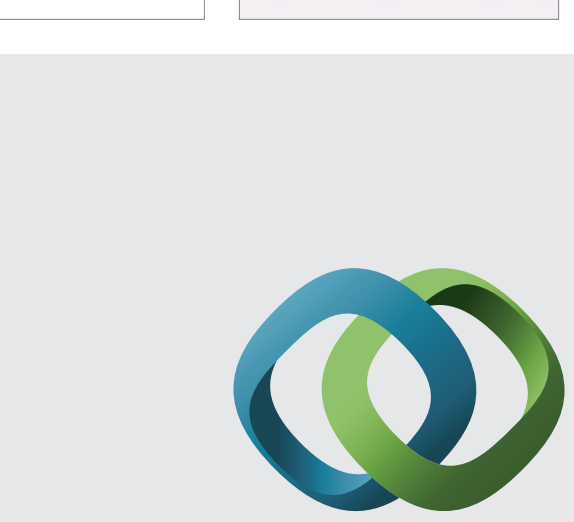

\section{Hindawi}

Submit your manuscripts at

http://www.hindawi.com
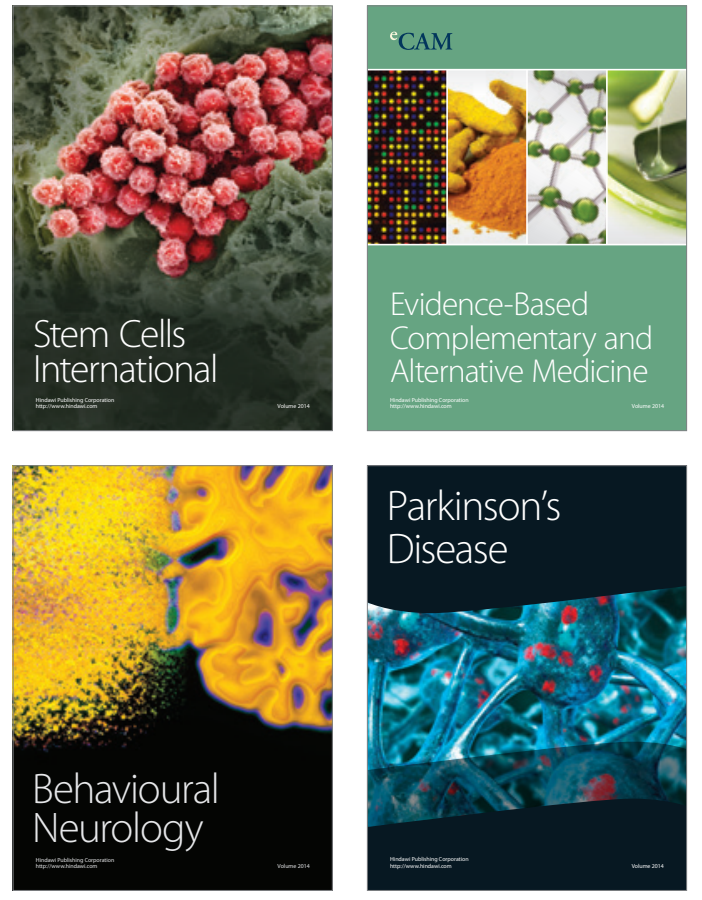
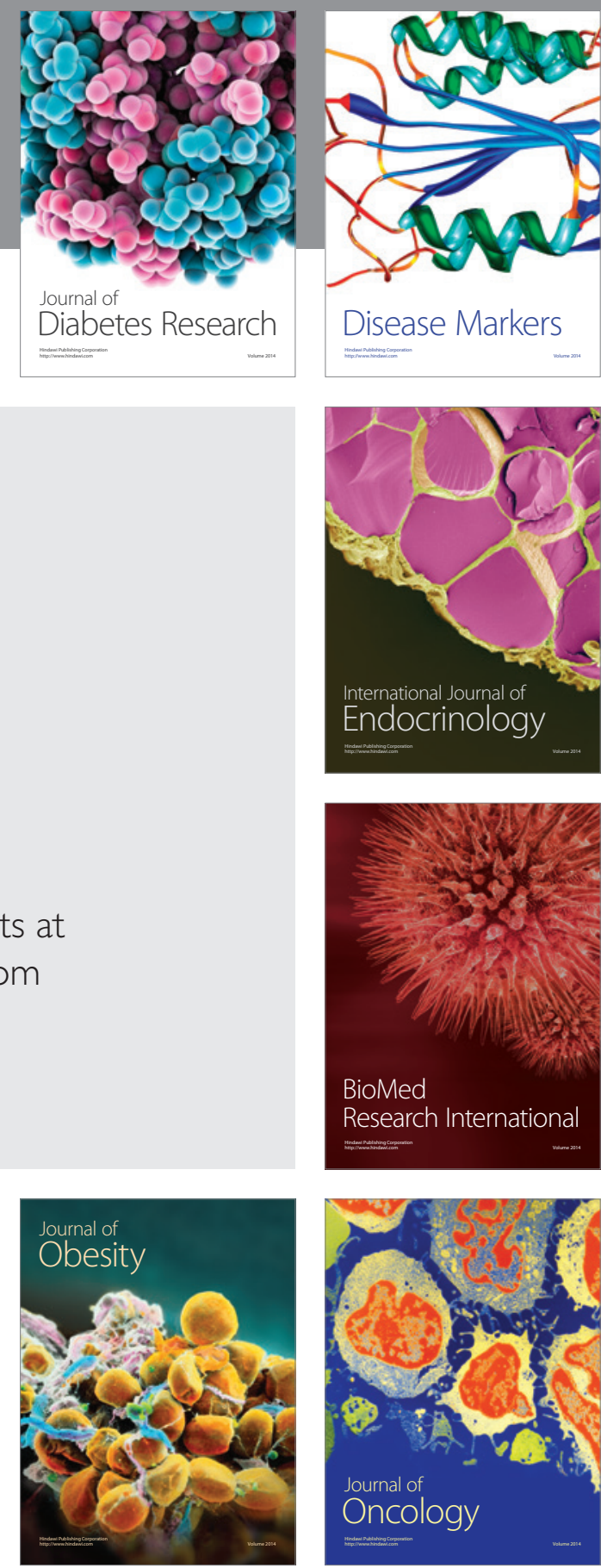

Disease Markers
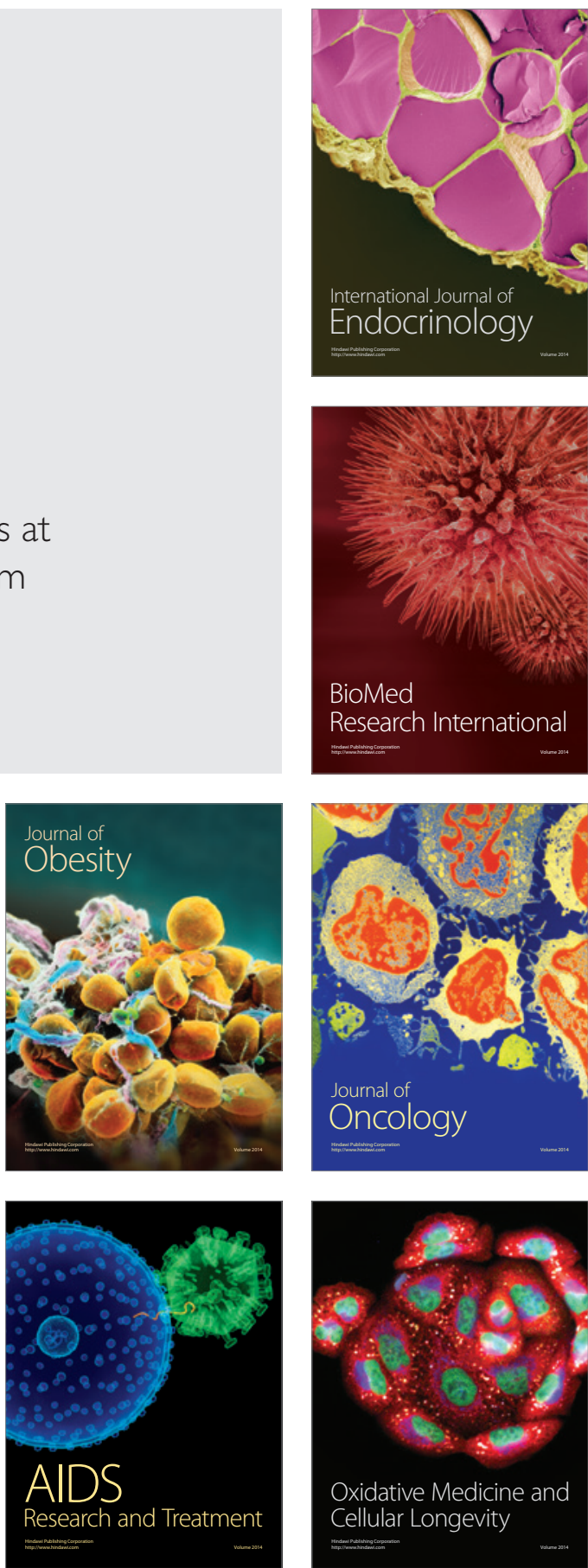